\title{
Caracterización y riesgo de fractura en población adulta chilena. Una nueva mirada a la ENS 2009-2010
}

\author{
Characterization and risk of fracture in Chile's adult \\ population. A new look at ENS 2009-2010
}

\author{
Donoso R, ${ }^{*}$ Margozzini $\mathrm{P},{ }^{\ddagger}$ Roman $\mathrm{J}^{\S}$
}

Pontificia Universidad Católica de Chile.

RESUMEN. Introducción: Las fracturas son lesiones relevantes en la vida de los individuos tanto por el costo del tratamiento, como por la carga en la vida laboral. La información en nuestro país acerca de esta patología es escasa, por lo que nos propusimos revisar datos epidemiológicos disponibles en la Encuesta Nacional de Salud (ENS). Material y métodos: Se calculó la prevalencia de vida de fracturas para la totalidad de la muestra y para los adultos mayores de 55 años, estimándose modelos de regresión logísticos distintos por sexo para la probabilidad de sufrir una fractura en la vida. Resultados: La prevalencia de vida de fracturas es de $18 \%$, con $25.1 \%$ para los adultos mayores de 55 años, observándose mayor prevalencia en hombres que en mujeres (22.5 y 13.8\% respectivamente). En el sexo masculino se asoció con fracturas: edad, tabaquismo, actividad física, consumo de alcohol e IMC, mientras que en el sexo femenino se asoció: edad, polifarmacia, estado civil y actividad física. Conclusión: Se encontró asociación entre la ocurrencia de fracturas y múltiples factores de riesgo conocidos para enfermedades crónicas, destacando la relevancia que poseen las fracturas en la población. Nueva evidencia orientada a estudiar este fenómeno se hace imperativa dado el envejecimiento progresivo de la población chilena.

Palabras clave: Fracturas, población, riesgo, género.
ABSTRACT. Introduction: Fractures are relevant injuries in the lives of individuals both for the cost of treatment and for the burden on working life. The information in our country about this pathology is scarce, so we set out to review epidemiological data available in the national health survey. Material and methods: The prevalence of fracture life was calculated for the entire sample and for adults over 55 years of age, with different logistic regression models estimated by sex for the likelihood of a fracture in life. Results: The prevalence of fracture life is $18 \%$, with $25.1 \%$ for adults over 55 years of age, with higher prevalence observed in men than in women (22.5\% and $13.8 \%$ respectively). In the male sex it was associated with fractures: age, smoking, physical activity, alcohol consumption, and BMI, while in the female sex it was associated: age, polypharmacy, marital status, and physical activity. Conclusion: An association was found between the occurrence of fractures and multiple known risk factors for chronic diseases, highlighting the relevance of fractures in the population. New evidence aimed at studying this phenomenon becomes imperative given the progressive ageing of the Chilean population.

Keywords: Fractures, population, risk, gender.

\section{Nivel de evidencia: IV}

\footnotetext{
* Magíster en Epidemiología.

* Departamento de Salud Pública.

${ }^{\S}$ Departamento de Traumatología y Ortopedia.
}

Facultad de Medicina de la Pontificia Universidad Católica de Chile. Chile.

Correspondencia:

Rodrigo Donoso MD, MS

Departamento de Traumatología y Ortopedia,

Facultad de medicina, Pontificia Universidad Católica de Chile. 


\section{Introducción}

En el ámbito mundial, tanto las lesiones como las enfermedades musculoesqueléticas son responsables de una alta carga para los sistemas de salud, siendo una causa importante de morbilidad y mortalidad. ${ }^{1}$ Éstas constituyen, dentro de las lesiones del aparato locomotor, 7.7\% de los años de vida ajustados por discapacidad (AVISA) de acuerdo con el estudio de carga de enfermedad del año 2007 en nuestro país, ${ }^{2}$ mientras que en el mundo el estudio Global Burden of Disease del año 2013 reveló que las fracturas presentan una incidencia anual que requerirá hospitalización de 290 por cada cien mil habitantes (38.5\% del total de lesiones por trauma anuales que requieren hospitalización), sumado a las de manejo ambulatorio que ascienden a 1,438 por cada cien mil habitantes (11.2\% del total de lesiones por trauma anuales manejadas de forma ambulatoria), todo esto sin considerar las fracturas que nunca llegan a consultarse en los servicios de salud.

Respecto a la realidad nacional, se ha observado un incremento progresivo en las altas hospitalarias por diagnóstico de fractura, independiente de su ubicación, observándose que en 1985 estos egresos constituyeron $3.3 \%$ del total, ${ }^{3}$ mientras que al año 2012 este ítem constituyó 4.8\% del total, ${ }^{4}$ lo que podría estar en línea con el envejecimiento progresivo de la población.

En este contexto, se han descrito múltiples factores de riesgo asociados con la probabilidad de sufrir alguna fractura a lo largo de la vida, dentro de los cuales se pueden destacar la edad, el sexo, el hábito tabáquico, el consumo de alcohol, el índice de masa corporal (IMC), la actividad física y el consumo de medicamentos, entre otros. ${ }^{5,6,7,8,9}$

Sin embargo, en nuestro país la información acerca de las fracturas en conjunto sigue siendo escasa. Basándonos en datos internacionales, se observa que las fracturas afectan a todos los segmentos etarios, con comportamientos diferentes de acuerdo con la edad y mecanismo lesional, ${ }^{10}$ observándose que podría constituir un fenómeno con una carga importante para la salud pública.

\begin{tabular}{|c|c|c|c|}
\hline \multirow[b]{2}{*}{ Edad } & \multirow{2}{*}{$\begin{array}{c}\text { Masculino } \\
\mathrm{n}(\%)\end{array}$} & \multirow{2}{*}{$\begin{array}{c}\text { Femenino } \\
\mathrm{n}(\%)\end{array}$} & \multirow{2}{*}{$\begin{array}{c}\text { Total } \\
\mathrm{n}(\%)\end{array}$} \\
\hline & & & \\
\hline$<20$ & $226(46.0)$ & 265 (54.0) & $491(9.1)$ \\
\hline $20-30$ & $317(40.0)$ & $476(60.0)$ & 793 (14.7) \\
\hline $30-40$ & 367 (41.2) & $524(58.8)$ & $891(16.5)$ \\
\hline $40-50$ & 397 (39.7) & 603 (60.3) & $1,000(18.5)$ \\
\hline $50-60$ & 364 (41.3) & 517 (58.7) & 881 (16.3) \\
\hline $60-70$ & $278(39.4)$ & $427(60.6)$ & 705 (13.0) \\
\hline $70-80$ & 187 (41.6) & 263 (58.4) & 450 (8.3) \\
\hline $80-90$ & 57 (31.7) & 123 (68.3) & $180(3.3)$ \\
\hline $90-100$ & 5 (23.8) & $16(76.2)$ & $21(0.4)$ \\
\hline Total & $2,198(40.6)$ & $3,214(59.4)$ & $5,412(100.0)$ \\
\hline
\end{tabular}

\begin{tabular}{|c|c|c|c|}
\hline & Masculino & Femenino & Total \\
\hline Edad & n (\%) & n (\%) & n (\%) \\
\hline$<20$ & $928,406(50.9)$ & $894,465(49.1)$ & 1'822,872 (13.8) \\
\hline $20-30$ & 1’207,721 (51.5) & 1'138,290 (48.5) & 2’346,012 (17.8) \\
\hline $30-40$ & $1^{\prime} 311,122$ (52.4) & 1'188,909 (47.6) & 2’500,032 (19.0) \\
\hline $40-50$ & $1 ' 139,796(45.4)$ & 1'372,857 (54.6) & $2 ’ 512,653(19.1)$ \\
\hline $50-60$ & $879,238(49.5)$ & $896,712(50.5)$ & $1 ’ 775,951(13.5)$ \\
\hline $60-70$ & $592,939(44.6)$ & 737,125 (55.4) & 1’330,064 (10.1) \\
\hline $70-80$ & $268,413(43.5)$ & 348,065 (56.5) & $616,479(4.7)$ \\
\hline $80-90$ & $88,418(36.0)$ & $157,411(64.0)$ & $245,830(1.9)$ \\
\hline $90-100$ & $3,711(13.7)$ & 23,426 (86.3) & $27^{\prime} 137(0.2)$ \\
\hline Total & $6 ’ 419,769(48.7)$ & 6’757,263 (51.3) & 13’177,033 (100.0) \\
\hline
\end{tabular}

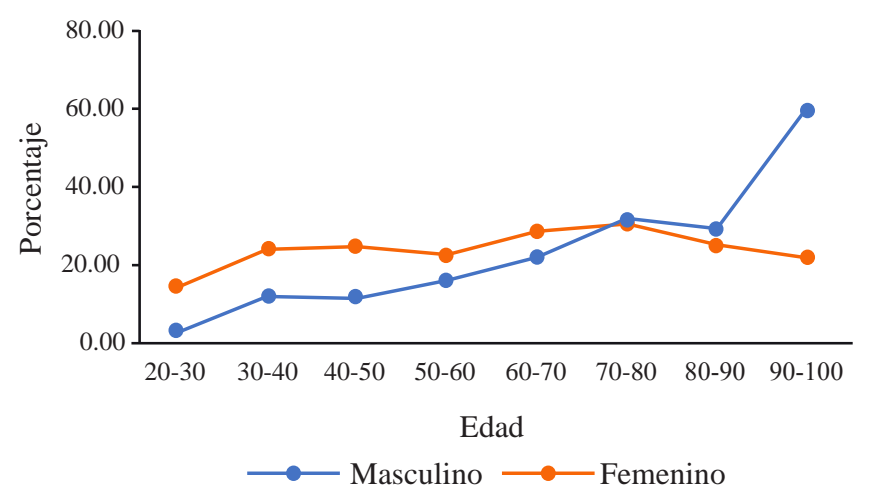

Figura 1: Prevalencia de vida de fracturas según edad y sexo.

Este estudio pretende identificar la distribución y el perfil de esta patología en la población adulta chilena en su totalidad y con un énfasis particular en la población mayor a 55 años, identificando los subgrupos de mayor riesgo en esta población. Esto podría contribuir a la implementación de nuevas políticas de promoción de salud y prevención de fracturas, destinando mayores recursos para el manejo de esta patología y sentando las bases para futuras investigaciones en el tema.

\section{Material y métodos}

La Encuesta Nacional de Salud (ENS) 2009-2010 (muestra aleatoria de hogares de tipo complejo (estratificada y multietápica por conglomerados) con representatividad nacional, regional y por zona rural y urbana incluyó, por primera vez, un módulo de osteoporosis que incorpora un cuestionario que indaga respecto a las fracturas en la población, incluyendo cuestionarios respecto al número de fracturas, sección del cuerpo afectado (respondido con base en imágenes del esqueleto), edad de las fracturas y el número de caídas durante el último año, todas estas por autorreporte.

En este contexto, el resultado principal de este estudio fue la prevalencia de vida de fracturas, calculada con base en el porcentaje de participantes que respondieron positi- 
vamente a la pregunta de haber sufrido una fractura a lo largo de su vida, considerándose un resultado secundario toda fractura reportada posterior a los 55 años. Este resultado secundario se calcula a partir de los 55 años para evitar la influencia protectora de osteoporosis que presentan las hormonas sexuales femeninas hasta antes de la menopausia. Las variables incluidas para el análisis fueron: edad, tipo de ocupación, nivel educacional en años, estado civil, hábito tabáquico, consumo semanal de alcohol, actividad física, antecedente de accidente vascular, antecedente de infarto agudo al miocardio, índice de masa corporal, uso de terapia hormonal de reemplazo (sólo en sexo femenino), grado de discapacidad, presencia de síntomas depresivos durante el último año, cantidad de medicamentos diarios consumidos y la macrozona geográfica en la cual se reside.

Se calculó la prevalencia de fracturas para toda edad, nivel educacional y región política de residencia, se estimó además la media de fracturas para toda edad y nivel educacional y la media de caídas durante los últimos 12 meses para toda edad, todas éstas separadas por sexo para efectos de comparación.

Se estima un modelo de regresión logística binaria stepwise, para el outcome principal descrito, ajustado por las variables propuestas, con el fin de ajustar sólo las variables que aportan significativamente al modelo. Se estiman en total tres modelos distintos: uno para la muestra completa

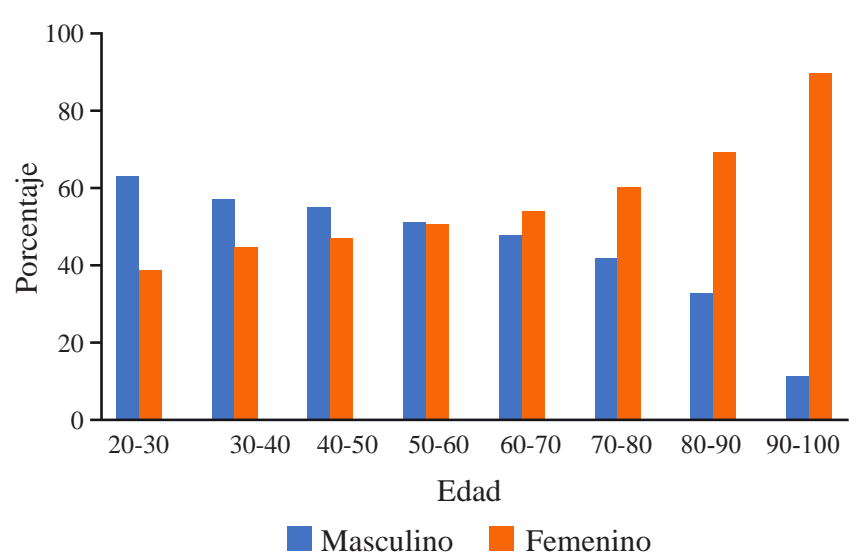

Figura 2: Peso relativo del sexo entre los individuos que reportan haber sufrido una fractura a toda edad.

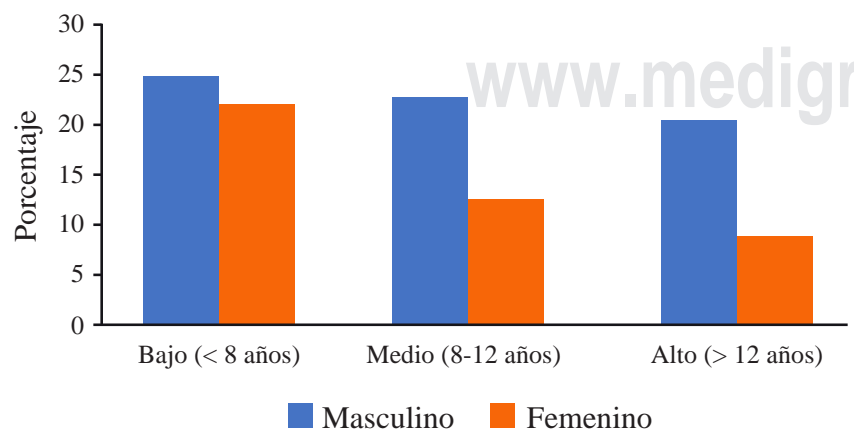

Figura 3: Prevalencia de fracturas según NEDU (nivel educacional) y sexo.

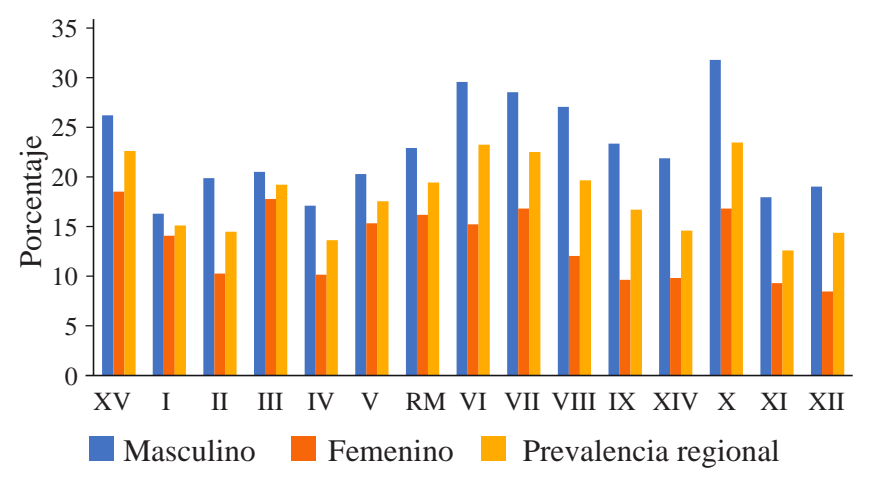

Figura 4: Prevalencia de vida de fracturas por región según sexo.

de individuos, otro para los mayores de 55 años con el resultadoprimario y el tercer modelo con base en el resultado secundario, sólo en el grupo de mayores de 55 años, estimando modelos independientes para ambos sexos.

Este estudio cuenta con la aprobación del comité de ética de la Pontificia Universidad Católica de Chile con dispensa de consentimiento informado por ser datos públicamente disponibles con consentimiento informado firmado para este fin al momento de la recolección de los datos. El análisis estadístico fue realizado utilizando el software SPSS $22^{\circledR}$.

\section{Resultados}

La muestra total consta de 5,412 individuos con datos válidamente emitidos y consentimiento informado firmado. Para el análisis de la submuestra de los adultos mayores de 55 años, el tamaño muestral fue de 1,839 individuos, de los cuales $45.3 \%$ fueron hombres y $54.7 \%$ mujeres; 167 hombres $(9.08 \%)$ y 299 mujeres (16.3\%) tenían edad $\geq 75$ años (Tablas 1 y 2).

Del total de la muestra, 880 individuos (que representan a aproximadamente 2,088,000 chilenos) reportan haber sufrido una fractura a lo largo de su vida, lo que da una prevalencia nacional de vida de fracturas de $18 \%$; mientras que para el subgrupo de adultos mayores de 55 años, el número de individuos que reportan una fractura es de 413 (que representan aproximadamente 796,000 chilenos), lo que corresponde a una prevalencia de vida de fracturas de $25.1 \%$ en este grupo etario (Tablas 1 y 2).

$\mathrm{Al}$ analizar la prevalencia de vida de fracturas según edad y sexo, se observa que el sexo masculino presenta una prevalencia relativamente estable para diferentes edades, mayor a la nacional a toda edad, mientras que el sexo femenino presenta un crecimiento constante a medida que la mujer va envejeciendo, superando a la prevalencia masculina en la tercera edad, observándose un punto de inflexión importante alrededor de los 80 años, momento desde el cual la prevalencia de fracturas aumenta considerablemente en las mujeres, alcanzando 59\% aproximadamente (Figura 1).

Sumado a esto, al observar el peso relativo que presenta cada sexo sobre la probabilidad de sufrir una fractura, se observan claras diferencias entre ambos, observándose que 
las fracturas son una patología reportada mayormente por hombres a edades más tempranas, invirtiéndose esta relación a partir de los 55 años (Figura 2).

Por otra parte, al observar la prevalencia de vida de fracturas según el nivel educacional, destaca que las mujeres de nivel educacional alto son las que reportan menos fracturas (8.9\%) significativamente en comparación con sus pares masculinos o con otros niveles educacionales (Figura 3).

Al comparar esta prevalencia entre las distintas regiones, destaca que la región con la mayor prevalencia de vida de fracturas es la de Los Lagos (X) y la menor la de Aysén (XI), observándose mayor prevalencia entre los individuos del sexo masculino respecto de individuos del sexo femenino independiente de la región (Figura 4).

Por otra parte, al considerar la media de fracturas, se observa una media nacional de 1.41 fracturas per cápita reportadas, destacando una media mayor en mujeres independientemente de la edad, exceptuando el grupo de menores de 20 años en que se observa una media mayor en el sexo masculino, sin diferencias significativas entre ambos sexos en este segmento etario (Figura 5).

La media de caídas autorreportadas en el último año es en promedio una caída per cápita en toda la población, revelándose una curva bimodal para el sexo masculino con un primer peak en la adultez joven que disminuye hacia los 40-50 años y un segundo peak a partir de los 70-80 años, no así para el sexo femenino en que se observa un crecimiento constante del número de caídas, superando a la media masculina en la tercera edad (Figura 6).

Al considerar sólo el grupo de adultos mayores de 55 años se observa un aumento en el número de caídas en ambos sexos, mientras que sólo en las mujeres aumenta la prevalencia de vida de fracturas y la prevalencia masculina se mantiene constante. De lo anterior, se desprende que a pesar de que ambos sexos aumentan su incidencia de caídas a mayor edad, éstas sólo conllevan un aumento en las fracturas en las mujeres.

Los modelos logísticos binarios para el outcome principal en la población total revelan que la edad incide significativamente en la prevalencia de fracturas independientemente del sexo. Es decir, a mayor edad mayor es la prevalencia de fracturas. En el modelo para hombres se asocia a presentar una fractura: el estatus de hábito tabáquico ( $\mathrm{p}=0.001)$, la

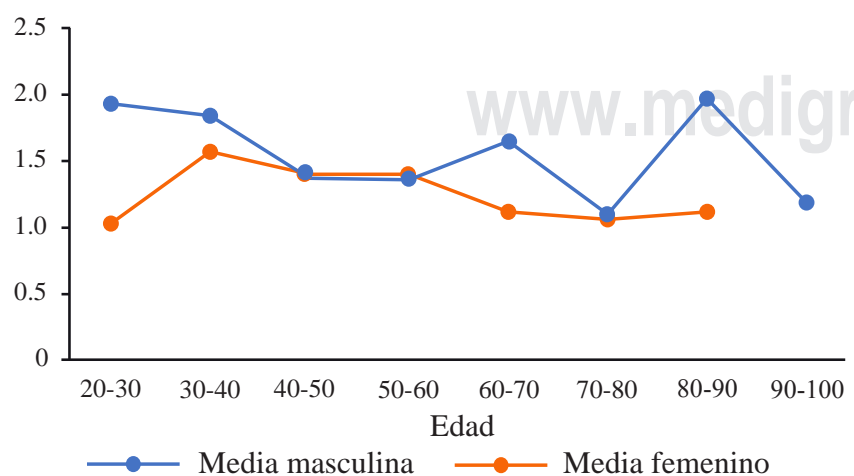

Figura 5: Media de fracturas durante la vida según edad y sexo.

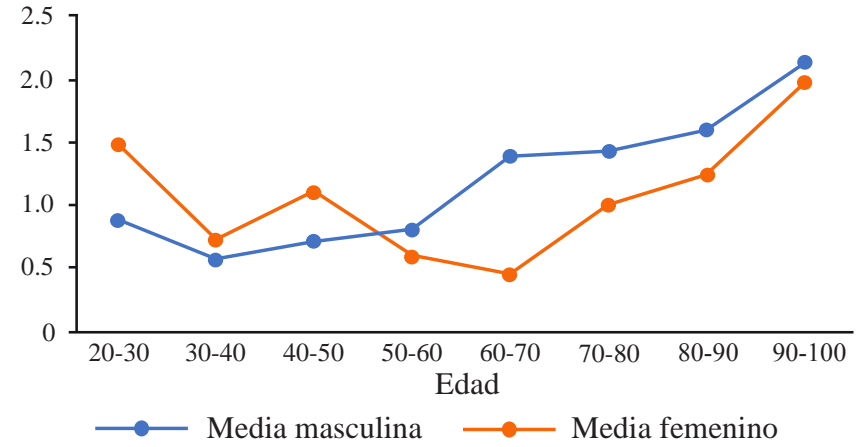

Figura 6: Media de caídas en el último año según edad y sexo.

actividad física ( $p=0.001$ ) y la edad ( $p<0.001$ ), observándose que las tres variables se comportan como factor de riesgo para este outcome (Tabla 3).

Por otra parte, en el modelo para las mujeres se asocia con la prevalencia de vida de fracturas, además de la edad (p $<0.001$ ): la cantidad de medicamentos que consume a diario $(p=0.016)$ y el estado civil $(p=0.043)$, observándose estas variables como factores de riesgo (Tabla 4).

En el caso de los modelos para el outcome principal sólo en los mayores de 55 años, destacan la actividad física ( $<$ < $0.001)$ y el consumo de alcohol semanal $(p=0.032)$ como factores de riesgo significativos en el caso del sexo masculino y la edad $(\mathrm{p}=0.022)$ como única variable significativa en el caso del sexo femenino, ambos modelos ajustados por nivel educacional (Tablas 5 y 6).

Por el contrario, al estimar el modelo en adultos mayores de 55 años, considerando sólo las fracturas reportadas después de los 55 años, se observa que, en el caso de los hombres, se asocia el IMC $(p=0.025)$ y el estado civil $(p<0.001)$ de manera significativa, mientras que en el caso de las mujeres, las variables asociadas significativamente son: la edad ( $\mathrm{p}<0.001)$, la cantidad de medicamentos $(p=0.022)$ y la actividad física $(p=0.014)$, ambos modelos ajustados por nivel educacional (Tablas 7 y 8).

\section{Discusión}

Nuestros resultados revelan la prevalencia de vida de fracturas, tanto para la totalidad de la población adulta chilena, como para los adultos mayores de 55 años utilizando la base de datos de la ENS 2009-2010, datos que previamente no se encuentran reportados en la literatura nacional o internacional para esta población. De esta manera, éste es el primer estudio en nuestro país basado en una muestra representativa de la población general que busca estimar la prevalencia de fracturas, basándose en que los individuos encuestados autorreporten su historial completo de fracturas, lo que ofrece la ventaja de capturar datos respecto a todas las fracturas que puede haber sufrido un individuo independiente del manejo que éstas hayan tenido. Esto permite el cálculo de la prevalencia de vida para la población general incluyendo tanto fracturas de manejo ambulatorio (muchas veces subreportadas por faltas de registros adecua- 
dos), como las de manejo quirúrgico, lo que permite dar una mirada más global al fenómeno.

En relación con la prevalencia de vida de fracturas, destaca el aumento de ésta que ocurre en la tercera edad, con mayor intensidad a partir de la octava década de vida, prevalencia que hasta ahora había permanecido desapercibida, al no existir estudios que la hubieran descrito en nuestra población. Uno de los hallazgos más relevantes de este estudio es la diferencia que presentan las fracturas en ambos sexos, así la prevalencia de vida de fracturas es mayor en el sexo masculino, pero, por otra parte, la media de fracturas es mayor en el sexo femenino, lo que comprueba que un porcentaje mayor de la población que se fractura son hombres, pero las mujeres que se fracturan lo hacen en promedio más veces que sus pares del sexo opuesto, poniendo a este grupo poblacional en un potencial mayor riesgo para esta patología. Esto cobra mayor relevancia al observar lo que ocurre entre los adultos mayores respecto a las fracturas y las caídas reportadas en los últimos 12 meses. En este grupo se observa que, a pesar de que ambos sexos aumentan su incidencia anual de caídas, sólo aumenta en mayor medida la prevalencia de fracturas del sexo femenino, mientras que, la prevalencia del sexo masculino se mantiene relativamente estable, observándose entonces que la mujer adulto mayor que se cae está en mayor riesgo potencial de sufrir una fractura respecto a sus pares del sexo masculino, todo esto teniendo en consideración que las fracturas generalmente obedecen a dos mecanismos etiológicos claros: aumentada fragilidad ósea y trauma relacionado a caídas, ${ }^{11}$ ambos mecanismos que juegan un rol importante en este grupo. Estos hallazgos son consistentes con múltiples estudios en la literatura internacional que datan incluso desde la década de los 60 en Europa y Asia, observándose patrones similares a los descritos en el presente estudio, ${ }^{12,13,14}$ siendo este el primer estudio del que tengamos conocimiento en nuestro medio que describe este fenómeno en la población general adulta chilena.

\begin{tabular}{|c|c|c|c|}
\hline \multicolumn{4}{|c|}{$\begin{array}{l}\text { Tabla 3: Modelo paso a paso, muestra } \\
\text { compleja, base total, sexo masculino. }\end{array}$} \\
\hline \multirow[b]{2}{*}{ Variable } & \multirow[b]{2}{*}{ OR } & \multicolumn{2}{|c|}{ IC $95 \%$} \\
\hline & & Inferior & Superior \\
\hline Edad & 1.03 & 1.014 & 1.046 \\
\hline \multicolumn{4}{|l|}{ Hábito tabáquico } \\
\hline Fumador activo & 2.435 & 1.522 & 3.893 \\
\hline Exfumador & 1.497 & 0.925 & 2.423 \\
\hline Nunca & 1 & - & - \\
\hline \multicolumn{4}{|l|}{ Actividad física } \\
\hline > 3 veces/semana & 3.407 & 1.769 & 6.562 \\
\hline 1-2 veces/semana & 2.177 & 0.954 & 4.971 \\
\hline$<1 \mathrm{vez} /$ semana & 2.032 & 1.078 & 3.828 \\
\hline No actividad física & 1 & - & - \\
\hline \multicolumn{4}{|l|}{ NEDU } \\
\hline Bajo & 1.651 & 0.804 & 3.391 \\
\hline Medio & 1.443 & 0.813 & 2.56 \\
\hline Alto & 1 & - & - \\
\hline
\end{tabular}

\begin{tabular}{|c|c|c|c|}
\hline \multirow[b]{2}{*}{ Variable } & \multirow[b]{2}{*}{ OR } & \multicolumn{2}{|c|}{ IC 95\% } \\
\hline & & Inferior & Superior \\
\hline Edad & 1.025 & 1.012 & 1.038 \\
\hline \multicolumn{3}{|l|}{$\begin{array}{l}\text { de medicamentos } \\
\text { Estado civil }\end{array}$} & 1.162 \\
\hline Casado & 2.191 & 1.179 & 4.07 \\
\hline Separado/divorciado & 2.096 & 1.095 & 4.014 \\
\hline Soltero & 1 & - & - \\
\hline \multicolumn{4}{|l|}{ NEDU } \\
\hline Bajo & 1.119 & 0.638 & 1.96 \\
\hline Medio & 1.007 & 0.621 & 1.634 \\
\hline Alto & 1 & - & - \\
\hline
\end{tabular}

Chile es un país en vías de desarrollo y es el primer país en América del Sur en ingresar a la Organización para la Cooperación y el Desarrollo (OCDE), hecho que lo ubica a la par de países desarrollados. Al comparar nuestros resultados con uno de estos países, como por ejemplo Inglaterra, se observan diferencias en relación con la prevalencia de vida de fracturas, la cual se estima en $38.2 \%$ para la población general, que al analizarla según sexo presenta una prevalencia de $44.7 \%$ para el sexo masculino y $32 \%$ para el sexo femenino, ${ }^{14}$ resultados mucho mayores a los encontrados en nuestra población. Esto obedecería al envejecimiento que presenta la población inglesa respecto a la chilena, que si bien este último presenta una población que está envejeciendo, la población inglesa se encuentra en una transición demográfica más avanzada, observándose en un estudio similar al nuestro que la población estudiada estaba conformada por $23 \%$ de hombres mayores de 75 años (2,782 individuos) y 31\% de mujeres mayores de 75 años (4,187 individuos) $)^{5}$ comparado con 9.08 y $16.3 \%$ respectivamente en nuestra muestra.

Este estudio revela, además, la asociación con distintas variables sociodemográficas y de hábitos respecto a la salud de los individuos, observándose diferencias en los modelos para ambos sexos. Aun así, se observa un determinante común entre ambos sexos que es la edad, factor de riesgo más importante para la ocurrencia de una fractura a lo largo de la vida. Dentro de las diferencias que determinan la prevalencia de vida de fracturas para cada sexo, se puede observar que la actividad física es el principal factor de riesgo significativo en los hombres junto con el hábito tabáquico, mientras que en las mujeres se observan asociaciones significativas con el estado civil y el número de medicamentos consumidos a diario, todos éstos en los modelos estimados para la población general. Cabe destacar que aún sin alcanzar significancia estadística se observa una tendencia al mayor riesgo de fracturas entre los individuos de niveles educacionales inferiores, sin importar del sexo, lo que puede obedecer a los distintos tipos de ocupaciones que desempeñan. . $^{4,15,16,17}$ 
Dentro de las variables estudiadas en los adultos mayores de sexo masculino, destaca el consumo de alcohol semanal como factor de riesgo significativo (OR: 1.046 [1.004-1.091]) por cada unidad de alcohol adicional consumida semanalmente. Kanis y colaboradores mostraron, con base en el análisis de individuos pertenecientes a tres cohortes (CaMos, DOES y el Rotterdam Study), que no había mayor riesgo para fracturas con ingestas menores a dos unidades diarias (una unidad definida en el reino unido como $8 \mathrm{~g}$ de alcohol puro), pero que por sobre este nivel se observa un mayor riesgo significativo para presentar cualquier tipo de fractura $(\mathrm{RR}=1.23$ [1.06-1.43]), una fractura osteoporótica $(\mathrm{RR}=1.38$ [1.16-1.65]) o en específico una fractura de cadera $\left(\mathrm{RR}=1.68\right.$ [1.19-2.36]), ${ }^{6}$ encontrando además un mayor riesgo no significativo entre individuos del sexo masculino respecto al femenino, lo que está en línea con nuestros resultados. Respecto a la actividad física, se puede cuestionar que los hábitos recabados por medio de una encuesta transversal no entreguen información respecto de la historia completa del individuo; aun así, estudios prospectivos en Tailandia han demostrado que para el caso de la actividad física, los hábitos respecto a éstos se mantienen relativamente estables a lo largo de la vida (contemplando desde la adultez joven hasta la adultez mayor), ${ }^{18}$ por lo que la actividad física reportada en la actualidad es una buena medida de la exposición al riesgo que ha tenido a lo largo de la vida el individuo que reporta seguir practicando deporte en la adultez mayor. En este contexto, la actividad física en el sexo masculino se comporta como factor de riesgo para presentar una fractura, lo que podría explicarse por medio de un mayor riesgo para trauma y caídas. ${ }^{11}$

En contraparte, el modelo estimado para el sexo femenino sólo revela una asociación significativa con la edad, observándose un OR de 1.027 (IC 95\%: 1.004-1.05), lo que puede obedecer a dos razones. En específico, estudios prospectivos en el Reino Unido entre los años 1988-1998 basados en la General Practice Research Database (GPRD), base de datos representativa de la distribución de la población nacional inglesa, estimó que, para el caso de pacientes mayores de 50 años, la incidencia anual estan-

\begin{tabular}{|c|c|c|c|}
\hline \multirow[b]{2}{*}{ Variable } & \multirow[b]{2}{*}{ OR } & \multicolumn{2}{|c|}{ IC $95 \%$} \\
\hline & & Inferior & Superior \\
\hline \multicolumn{4}{|l|}{ Actividad física } \\
\hline > 3 veces/semana & 13.71 & 4.237 & 44.359 \\
\hline 1-2 veces/semana & 2.236 & 0.408 & 12.264 \\
\hline$<1 \mathrm{vez} / \mathrm{semana}$ & 5.296 & 1.271 & 22.073 \\
\hline No actividad física & 1 & - & - \\
\hline Consumo de alcohol semanal & 1.046 & 1.004 & 1.091 \\
\hline Edad & 1.013 & 0.976 & 1.052 \\
\hline \multicolumn{4}{|l|}{ NEDU } \\
\hline Bajo & 1.722 & 0.636 & 4.658 \\
\hline Medio & 1.434 & 0.513 & 4.009 \\
\hline Alto & 1 & - & - \\
\hline
\end{tabular}

\begin{tabular}{|c|c|c|c|}
\hline \multicolumn{4}{|c|}{$\begin{array}{l}\text { Tabla 6: Modelo paso a paso, muestra } \\
\text { compleja, adultos > 55, sexo femenino. }\end{array}$} \\
\hline \multirow[b]{2}{*}{ Variable } & \multirow[b]{2}{*}{ OR } & \multicolumn{2}{|c|}{ IC $95 \%$} \\
\hline & & Inferior & Superior \\
\hline Edad & 1.027 & 1.004 & 1.05 \\
\hline \multicolumn{4}{|l|}{ NEDU } \\
\hline Bajo & 1.268 & 0.542 & 3.07 \\
\hline Medio & 1.216 & 0.49 & 3.019 \\
\hline Alto & 1 & - & - \\
\hline
\end{tabular}

darizada para cualquier fractura es de 174.1/10,000 años-persona en el caso de las mujeres, respecto a un 78.1/10,000 años-persona en el caso de los hombres, ${ }^{10}$ demostrando que en este grupo etario las mujeres representan un grupo especial de riesgo para esta patología. Además, un mecanismo importante de fracturas en este segmento etario es la osteoporosis, definida como una enfermedad sistémica del hueso caracterizada por baja densidad ósea y pérdida de su microarquitectura trabecular, lo que se objetiva en la densitometría ósea como un $\mathrm{T}$ score $<-2.5$ desviaciones estándar. ${ }^{19}$ Así, en el caso del sexo femenino se han reportado pérdidas de hasta 35-50\% de hueso trabecular y 25-30\% de hueso cortical desde la perimenopausia y a medida que envejece la persona, comparado con una pérdida aproximada de 15-45\% y 5-15\% respectivamente en el caso del sexo masculino. ${ }^{19}$ Más aún, respecto a las caídas durante el último año reportadas en este estudio, se observa que en la tercera edad las mujeres presentan una media de caídas mayor que sus pares masculinos, a pesar del incremento considerable de este evento en ambos sexos. Así, la ENS 2009-2010 no incorporó exámenes que permitieran estimar el riesgo de osteoporosis en estas casos, no contando con densitometrías óseas ni con niveles de vitamina $\mathrm{D}$, micronutriente con demostrada deficiencia en mujeres chilenas postmenopáusicas sanas con adecuada exposición solar (60\% comparado con $27 \%$ en mujeres de similares características premenopáusicas) ${ }^{20}$ y más aún en pacientes que han sufrido una fractura de cadera en quienes el déficit puede alcanzar cifras de alrededor de $98 \%$ en población que se atiende en la red de salud UC-Christus como demostraron Schweitzer y colaboradores ${ }^{21}$ datos que serían relevantes de medir en próximas versiones de esta encuesta para poder evaluar de mejor manera el riesgo que presentan las pacientes de la tercera edad respecto a las fracturas.

La ENS incluye una pregunta que busca esclarecer a qué edad los encuestados sufrieron las fracturas autorreportadas, por lo que para evaluar el posible sesgo de memoria que se puede presentar entre los adultos mayores al tener que recordar fracturas que pueden haber ocurrido hace muchos años, se estimó un modelo sólo para las fracturas reportadas después de los 55 años. En el caso del sexo masculino, se observa una asociación significativa con el estado civil y el IMC, ajustado por edad y NEDU. Así, en el caso del estado civil se observa un efecto protector significativo del soltero respecto al casado, lo que está en concordancia con estudios 
realizados en Inglaterra que emplean una metodología similar a la del presente estudio para estimar modelos en la tercera edad. ${ }^{5}$ Además, al observar la variable IMC, se observa que un exceso de peso es protector significativamente respecto a un IMC considerado bajo $(<18.5)$ o normal (18.525) (OR: 0.194 [0.05-0.747]), lo que se explicaría por un potencial efecto protector del exceso de masa adiposa sobre la densidad mineral ósea (síntesis de estrógeno extragonadal) y la protección física contra impactos que ofrece esta masa, ${ }^{22}$ efecto que se perdería en el caso de la obesidad mórbida debido a que la relación de la densidad mineral ósea con el IMC no es lineal y presentaría un umbral por sobre el cual no hay beneficios en este aspecto, además de un riesgo agregado para caídas en esta población debido a la sarcopenia y pérdida de fuerza asociados, ${ }^{22}$ lo que se observa por una tendencia no significativa al mayor riesgo en el grupo de obesos mórbidos.

Por otra parte, en el modelo estimado para el sexo femenino, se observa una asociación significativa con la edad, la actividad física y la cantidad de medicamentos que consumen, destacando que la actividad física actúa como un factor protector en este grupo, significativo sólo en el caso de practicar la actividad más de tres veces por semana (OR: 0.078 [0.015-0.413]), lo que está en línea con la literatura que ubica al ejercicio como una estrategia de prevención, tanto primaria como secundaria, para la fractura de cadera en los adultos mayores..$^{22}$ Además, el ejercicio en este grupo, en especial el entrenamiento con resistencia progresiva (PRT, por sus siglas en inglés) logra ganancias clínicamente significativas de masa muscular, fuerza y capacidad aeróbica en adultos mayores frágiles, tanto hombres como mujeres, hasta los 96 años inclusive. ${ }^{7}$ Respecto al uso de medicamentos, se ha definido como polifarmacia el consumo de cinco o más medicamentos, en este contexto cada medicamento adi-

\begin{tabular}{|c|c|c|c|}
\hline \multirow[b]{2}{*}{ Variable } & \multirow[b]{2}{*}{ OR } & \multicolumn{2}{|c|}{ IC 95\% } \\
\hline & & Inferior & Superior \\
\hline \multicolumn{4}{|l|}{ IMC } \\
\hline Bajo o normal & 1 & - & - \\
\hline Sobrepeso & 0.397 & 0.11 & 1.431 \\
\hline Obeso & 0.194 & 0.05 & 0.747 \\
\hline Obeso mórbido & 2.269 & 0.346 & 14.889 \\
\hline \multicolumn{4}{|l|}{ Estado civil } \\
\hline Casado & 1 & - & - \\
\hline Separado/divorciado & 0.572 & 0.194 & 1.687 \\
\hline Soltero & 0.015 & 0.003 & 0.081 \\
\hline Edad & 1.062 & 0.999 & 1.128 \\
\hline \multicolumn{4}{|l|}{ NEDU } \\
\hline Bajo & 0.87 & 0.148 & 5.104 \\
\hline Medio & 0.675 & 0.091 & 4.992 \\
\hline Alto & 1 & - & - \\
\hline
\end{tabular}

$\mathrm{IMC}=$ índice de masa corporal; NEDU = nivel educacional.

\begin{tabular}{|c|c|c|c|}
\hline \multirow[b]{2}{*}{ Variable } & \multirow[b]{2}{*}{ OR } & \multicolumn{2}{|c|}{ IC $95 \%$} \\
\hline & & Inferior & Superior \\
\hline Edad & 1.065 & 1.039 & 1.092 \\
\hline \multicolumn{4}{|l|}{ Actividad física } \\
\hline > 3 veces/semana & 0.078 & 0.015 & 0.413 \\
\hline 1-2 veces/semana & 1.904 & 0.578 & 6.27 \\
\hline$<1 \mathrm{vez} /$ semana & 0.534 & 0.053 & 5.336 \\
\hline No actividad física & 1 & - & - \\
\hline Cantidad de medicamentos & 1.116 & 1.016 & 1.226 \\
\hline \multicolumn{4}{|l|}{ NEDU } \\
\hline Bajo & 0.974 & 0.312 & 3.043 \\
\hline Medio & 0.912 & 0.279 & 2.988 \\
\hline Alto & 1 & - & - \\
\hline
\end{tabular}

cional presenta un mayor riesgo de fractura posterior a los 55 años en nuestro modelo (OR: 1.116 [1.016-1.226]), lo que se ha observado en estudios recientes en adultos mayores, en los cuales el mayor uso de medicamentos, en especial la polifarmacia, se asocia significativamente con un mayor número de caídas en los últimos seis meses ( $\mathrm{r}=0.164$, $\mathrm{p}=$ 0.001 ), siendo esta relación aún mayor en el caso del sexo femenino $(r=0.257),{ }^{8}$ lo que está en línea con nuestros hallazgos a la vez que explica el mecanismo por el cual el mayor consumo de medicamentos aumentaría el riesgo de sufrir cualquier fractura durante la tercera edad. ${ }^{8}$

Dentro de las limitaciones del presente estudio, podemos comentar que, al ser un estudio observacional, existe la posibilidad de que haya variables no medidas que estén explicando las asociaciones encontradas. Otro punto importante para comentar es el potencial sesgo de memoria, ya que se le está pidiendo al paciente que recuerde todas las fracturas que ha tenido en su vida. Si bien este podría presentar un sesgo de información importante, estudios prospectivos que han buscado validar el autorreporte de una fractura contra el historial clínico o reportes de radiología han demostrado una buena correlación entre ambas (kappa: 0.77-0.89) y aproximadamente $11 \%$ de falsos positivos, ${ }^{23,24}$ lo que no descarta la posibilidad de que se recuerden las fracturas más recientes y no las que han ocurrido con mucha anterioridad, junto con un potencial subreporte de las fracturas asintomáticas o que no hayan buscado atención médica. Cabe destacar una posible exclusión de los adultos mayores que no estuvieran en condiciones de responder o se encontraran institucionalizados. En estos casos, se entrevistó a un proxy familiar cercano, por lo que nuestro estudio puede no estar estimando la prevalencia exacta en este grupo. Por último, los adultos mayores a 75 años son considerados supervivientes, por lo que podrían representar una población excepcionalmente sana respecto a factores de riesgo y comorbilidades, lo que podría implicar un sesgo en la validez de nuestros hallazgos en este grupo. 
Consideramos que es el primer estudio en población general respecto al tema, lo que puede sentar las bases para futuras investigaciones en una patología que no ha recibido la atención que requiere por parte de la salud pública de nuestro país. En este contexto, nuevas versiones de la ENS que incluyan módulos más detallados respecto a las fracturas y sus determinantes, en especial mediciones orientadas a determinar el estado de osteoporosis de la población (densitometrías óseas y niveles de vitamina $\mathrm{D}$, entre otros), con especial énfasis en los adultos mayores, se hace imperativo. Urge mejorar la capacidad de atención preventiva y geriátrica del país. En esta misma línea, la pesquisa de déficit de vitamina $\mathrm{D}$ no está priorizada como meta de salud pública, posiblemente por la escasez de información que existe respecto al estado en la población general de este micronutriente; así, políticas públicas que fijaran metas de vitamina D para la población y una mayor destinación de recursos para el estudio podrían contribuir a la generación de medidas preventivas para las fracturas. Aun así, nuestro estudio da luces de algunos de los factores sociodemográficos y hábitos de vida modificables que se asocian con la prevalencia de vida de fracturas. Futuras investigaciones podrían contribuir a mejorar el conocimiento de los determinantes de tan compleja patología que afecta transversalmente a toda la población.

\section{Conclusión}

Se demuestra la relevancia de las fracturas para la población chilena, se encontró asociación entre la ocurrencia de fracturas y múltiples factores de riesgo conocidos para enfermedades crónicas, destacando la relevancia que poseen las fracturas en la población. Nueva evidencia orientada a estudiar este fenómeno se hace imperativa dado el envejecimiento progresivo de la población chilena.

\section{Referencias}

1. Haagsma JA, Graetz N, Bolliger I, Naghavi M, Higashi H, Mullany $\mathrm{EC}$, et al. The global burden of injury: incidence, mortality, disabilityadjusted life years and time trends from the Global Burden of Disease study 2013. Inj Prev. 2016; 22(1): 3-18.

2. Bedregal P, Margozzini P, González C. Informe final estudio de carga de enfermedad y carga atribuible [Internet]. 2007. Disponible en: https://medicina.uc.cl/wp-content/uploads/2018/08/Informe_final_ estudio_carga_2007.pdf

3. Contreras L, Kirschbaum A, Pumarino H. Epidemiologia de las fracturas en Chile. Rev Med Chil. 1991; 119: 92-8.

4. Ministerio de Salud. Base de datos de egresos hospitalarios de Chile. 2012.
5. Scholes S, Panesar S, Shelton NJ, Francis RM, Mirza S, Mindell JS, et al. Epidemiology of lifetime fracture prevalence in England: a population study of adults aged 55 years and over. Age Ageing. 2014; 43(2): 234-40.

6. Kanis JA, Johansson H, Johnell O, Oden A, De Laet C, Eisman JA, et al. Alcohol intake as a risk factor for fracture. Osteoporos Int. 2005; 16(7): 737-42.

7. Fiatarone MA, Marks EC, Ryan ND, Meredith CN, Lipsitz LA, Evans WJ. High-intensity strength training in nonagenarians. Effects on skeletal muscle. JAMA. 1990; 263 (22): 3029-34.

8. Wong H, Heuberger R, Logomarsino J, Hewlings S. Associations between alcohol use, polypharmacy and falls in older adults. Nurs Older People. 2016; 28(1): 30-6.

9. Oyen J, Gram Gjesdal C, Nygard OK, Lie SA, Meyer HE, Apalset EM, et al. Smoking and body fat mass in relation to bone mineral density and hip fracture: the Hordaland Health Study. PLoS One. 2014; 9(3): e92882. Erratum in: PLoS One. 2014; 9(6): e101335.

10. van Staa TP, Dennison EM, Leufkens HG, Cooper C. Epidemiology of fractures in England and Wales. Bone. 2001; 29(6): 517-22.

11. Cumming RG, Nevitt MC, Cummings SR. Epidemiology of hip fractures. Epidemiol Rev. 1997; 19(2): 244-57.

12. Buhr AJ, Cooke AM. Fracture patterns. Lancet. 1959; 1(7072): 531-6.

13. Wong PC. Fracture epidemiology in a mixed southeastern Asian community (Singapore). Clin Orthop Relat Res. 1966; 45: 55-61.

14. Donaldson LJ, Reckless IP, Scholes S, Mindell JS, Shelton NJ. The epidemiology of fractures in England. J Epidemiol Community Health. 2008; 62(2): 174-80.

15. Dong XS, Wang X, Largay JA. Occupational and nonoccupational factors associated with work-related injuries among construction workers in the USA. Int J Occup Environ Health. 2015; 21(2): 142-50.

16. Kim SK, Kim H, Lee K, Kang HT, Oh SS, Ko SB. The Relationship between Injury and Socioeconomic Status in Reference to the Fourth Korean National Health and Nutrition Examination Survey. Ann Occup Environ Med. 2014; 26(1): 1.

17. Montano D. Upper body and lower limbs musculoskeletal symptoms and health inequalities in Europe: an analysis of cross-sectional data. BMC Musculoskelet Disord. 2014; 15: 285.

18. Friedman HS, Martin LR, Tucker JS, Criqui MH, Kern ML, Reynolds CA. Stability of physical activity across the lifespan. J Health Psychol. 2008; 13(8): 1092-104.

19. Francis RM. Falls and fractures. Age Ageing. 2001; 30 Suppl 4: 25-8.

20. González G. Vitamin D status among healthy postmenopausal women in South America. Dermatoendocrinol. 2013; 5(1): 117-20.

21. Schweitzer D, Amenábar PP, Botello E, López M, Saavedra Y, Klaber I. Prevalencia de insuficiencia y deficiencia de vitamina $\mathrm{D}$ en adultos mayores con fractura de cadera en Chile. Rev Med Chil [Internet]. 2016; 144(2): 175-80. Disponible en: http://www.scielo.cl/scielo. php?script $=$ sci_arttext\&pid=S0034-98872016000200005\&lng $=$ en\&n $\mathrm{rm}=$ iso\&tlng=en

22. Scott D, Daly RM, Sanders KM, Ebeling PR. Fall and fracture risk in sarcopenia and dynapenia with and without obesity: the role of lifestyle interventions. Curr Osteoporos Rep. 2015; 13(4): 235-44.

23. Siqueira FV, Facchini LA, Hallal PC. The burden of fractures in Brazil: a population-based study. Bone. 2005; 37(2): 261-6.

24. Ismail AA, O'Neill TW, Cockerill W, Finn JD, Cannata JB, Hoszowski K, et al. Validity of self-report of fractures: results from a prospective study in men and women across Europe. EPOS Study Group. European Prospective Osteoporosis Study Group. Osteoporos Int. 2000; 11(3): 248-54. 